\title{
Produção de material didático durante a pandemia da Covid-19: correlações entre anatomia e procedimentos clínicos
}

\author{
Production of teaching material during the Covid-19 pandemic: correlation between \\ anatomy and clinical procedures \\ Producción de material didáctico durante la pandemia Covid-19: correlaciones entre \\ anatomía y procedimientos clínicos
}

João Lucas Nunes de Moura1', Breno Alcará Ferreira1', Guilherme Okoti de Melo', Ligia Harumi Vilela Bartnick Tanaka1, Giovana Geraldini Chermont'1, Flávio de Paula Moraes¹, Luana Rossato $^{1}$, Silvia Cristina Figueira Olinto ${ }^{1 *}$.

\section{RESUMO}

Objetivo: Descrever as etapas para a produção de material didático anatomoclínico por alunos do curso de Medicina no período da pandemia da COVID-19. Relato de experiência: O material didático correlacionou pontos de referência e/ou vias de acesso de estruturas anatômicas abordadas no $1^{\circ}$ semestre do curso de medicina com o contexto das práticas médicas cirúrgicas. A elaboração do material envolveu duas etapas: 1ㅇ: revisão dos tópicos teórico-práticos da Anatomia I, 2ํ: seleção de imagens cadavéricas, artigos científicos, casos clínicos e questões relacionadas a cada procedimento cirúrgico dos tópicos: cabeça, pescoço, tórax, membros superiores e inferiores. A produção do material de ensino gerou uma apostila teórico-prática com descrições detalhadas de cada procedimento, imagens sequenciais com legendas das regiões de referência ou vias de acesso necessárias para a realização do procedimento ou técnica cirúrgica, casos clínicos com questões norteadoras acerca do procedimento e/ou estrutura anatômica. Considerações finais: O material didático desenvolvido complementará os estudos teórico-práticos da disciplina Anatomia Humana e Cirúrgica, proporcionando em um único material correlações da ciência Anatomia com a futura prática do profissional médico.

Palavras-chave: Material didático, Anatomia, Procedimentos cirúrgicos.

\begin{abstract}
Objective: To describe the steps for the production of anatomoclinical teaching material by medical students during the COVID-19 pandemic period. Experience report: The teaching material correlated reference points and/or access routes of anatomical structures addressed in the 1st semester of the medical course with the context of medical medical practices. The preparation of the material involved two steps: 1 st: review of the theoretical-practical Anatomy I, 2nd: selection of cadaveric images, scientific articles, clinical cases and questions related to each surgical procedure of the methods: head, neck, chest, upper limbs and lower. The production of the teaching material generated a theoretical-practical booklet with descriptions of each procedure, sequential images with captions of the reference regions or access routes for carrying out the procedure or surgical technique, clinical cases with guiding questions about the procedure and/or anatomical structure. Final considerations: The didactic material developed will complement the theoretical-practical studies of the Human and Surgical Anatomy discipline, providing in a single material correlation of the Anatomy science with the future practice of the medical professional.
\end{abstract}

Keywords: Teaching material, Anatomy, Surgical procedures.

1 Universidade Federal da Grande Dourados (UFGD), Dourados - MS. *E-mail: silviafigueira@ufgd.edu.br

PUBLICADO EM: 8/2021 


\section{RESUMEN}

Objetivo: Describir las etapas de producción de material didáctico anatómico-clínico por estudiantes de medicina durante el período pandémico del COVID-19. Informe de experiencia: El material didáctico correlacionó los puntos de referencia y / o vías de acceso de las estructuras anatómicas abordadas en el 1er semestre de la carrera de Medicina con el contexto de la práctica médica médica. La elaboración del material contó con dos pasos: 10: revisión teórico-práctica de Anatomía I, 20: selección de imágenes de cadáveres, artículos científicos, casos clínicos y cuestiones relacionadas con cada procedimiento quirúrgico de los métodos: cabeza, cuello, tórax, cabeza de extremidades e inferiores. La producción del material didáctico generó un cuadernillo teórico-práctico con descripciones de cada procedimiento, imágenes secuenciales con leyendas de las regiones de referencia o vías de acceso para realizar el procedimiento o técnica quirúrgica, casos clínicos con preguntas orientadoras sobre el procedimiento y/o estructura anatómica. Consideraciones finales: El material didáctico desarrollado complementará los estudios teórico-prácticos de la disciplina de Anatomía Humana y Quirúrgica, aportando en un solo material correlaciones entre la ciencia de la Anatomía y la futura práctica del profesional médico.

Palabras clave: Material didáctico, Anatomía, Procedimientos quirúrgicos.

\section{INTRODUÇÃO}

O conhecimento do corpo humano, através dos tempos, sempre exerceu fascínio sobre o homem. Entender como somos formados, a constituição interna e as diferenças entre os sexos, sempre estimulou e continuará estimulando a curiosidade humana. Os primeiros registros históricos de dissecações humanas realizadas com finalidade educativa, dentro de um contexto ensino-aprendizagem, datam ainda do século III a.C. (ALBUQUERQUE JDS, et al., 2020).

Com o passar dos séculos, artistas como Galeno, Leonardo da Vinci, Michelangelo dentre outros também colaboraram, cada um ao seu tempo, para o seu desenvolvimento da ciência Anatomia. Andreas Versallius, um anatomista belga, em 1539 demonstrou, através de dissecações em corpos humanos, que as descrições anatômicas de Galeno não se referiam a dissecações realizadas na espécie humana, corrigindo os equívocos que prevaleciam desde a Antiguidade, e através de sua contribuição recebeu o título de "pai da anatomia cientifica moderna", dividindo a Anatomia em antes e depois dos estudos do autor (TALAMONI ACB e BERTOLLI FILHO C, 2014).

Segundo Salbego C, et al. (2015), para qualquer profissional da área da saúde é fundamental um adequado conhecimento da ciência Anatomia, uma vez que esta proporciona a compreensão para os estudos quanto à organização, morfologia, localização bem como a função dos órgãos que em conjunto constituirão os sistemas observados no ser humano.

De maneira geral, o ensino da disciplina é realizado nas universidades através de fundamentações teóricas (geralmente aulas expositivas) e práticas (aulas no laboratório de Anatomia). A abordagem prática permite a visualização tridimensional da forma, percepção da organização e textura, bem como a localização dos órgãos e suas inter-relações com as demais estruturas que constituem o ser humano, porém a dificuldade na aquisição de cadáveres não reclamados é uma realidade nas instituições de ensino superior (KARBASI Z e NIAKAN KALHORI SR, 2020; SALBEGO C, et al., 2015).

Contudo, a estrutura exigida a partir de 2014 pelas Novas Diretrizes Curriculares Nacionais para o Curso de Medicina, demandaram que as faculdades implementassem adequações curriculares. Alterações como aumento na duração do internato e no ensino de matérias clínicas, além do debate da humanização profissional e inserção do aluno no Sistema Único de Saúde foram implementadas, fazendo com que disciplinas do ciclo básico tivessem suas cargas horárias reduzidas e conteúdos aglutinados (CASTRO KS, et al., 2021).

A disciplina de Anatomia foi particularmente atingida por este contexto, exigindo que o plano de ensino priorizasse apenas os pontos principais de abordagem, bem como elaborasse estratégias mais produtivas e 
cativantes em sala de aula. Ademais, esta dinâmica coeriu os acadêmicos a aprender e memorizar o conteúdo em menor intervalo de tempo, acarretando maiores taxas de reprovações e inseguranças quanto ao conhecimento adquirido (CASTRO KS, et al., 2021).

Dessa forma, a dificuldade na aquisição de material cadavérico, as inúmeras estruturas anatômicas para o estudo e a escassez de material didático complementar voltado a temática: correlações entre a estrutura anatômica e procedimentos / técnicas utilizadas na prática médica, nos remete a pensar sobre a necessidade da inclusão de diferentes ferramentas educacionais para contribuição no processo de ensino-aprendizagem sobre a disciplina (CABRAL RH, MASUKO TS,2020)

Ademais, com a pandemia de COVID-19 declarada pela Organização Mundial de Saúde em 11 de março de 2020, diversos setores da sociedade tiveram seu funcionamento afetado, dentre eles a educação. Com 0 fechamento de escolas e universidades, o ensino, atividades e eventos passaram a ser executados de modo remoto. Esta conjuntura se agrava na disciplina de Anatomia Humana pela demanda inerente de aulas práticas, dificultando a sedimentação da matéria aprendida (MELLO JM, et al., 2010). Portanto, estratégias que visem melhorar a assimilação do conteúdo anatômico e que se integrem às aulas expositivas em regime virtual são imprescindíveis (GOMES VTS, et al., 2020).

Tendo em vista as dificuldades do processo ensino-aprendizagem no tocante à Anatomia Humana no contexto da pandemia da COVID-19 e a importância da oferta de material complementar, o presente trabalho teve como objetivo relatar a experiência de produção de material didático a ser aplicado no curso de Medicina, visando oferecer alternativas complementares para implementar o estudo da disciplina Anatomia Humana e Cirúrgica. Para tal, foi elaborado um material didático complementar sobre a correlação do estudo de determinadas estruturas ou regiões anatômicas para a realização de procedimentos ou técnicas voltadas a prática clínica.

\section{RELATO DE EXPERIÊNCIA}

Trata-se de um estudo descritivo, do tipo relato de experiência, realizado por acadêmicos do terceiro semestre de um curso de Medicina de uma universidade federal localizada na região centro-oeste do Brasil. Em virtude da pandemia da COVID-19, houve a paralização das aulas presenciais em todo o mundo. No intuito de correlacionar estruturas bem como vias de acesso com a prática cirúrgica, foi elaborado um material de ensino complementar a ser utilizado pelas disciplinas de Anatomia Humana e Anatomia Cirúrgica. A produção do material didático ocorreu de forma online em duas etapas:

Na primeira etapa foi realizada uma revisão e discussão dos seguintes tópicos teórico-práticos que são abordados na disciplina de Anatomia Humana I: Cabeça, pescoço, tórax, membros inferiores e superiores. Para esses assuntos, destacou-se estruturas e vias de acesso que são importantes para a realização de procedimentos cirúrgicos.

Em seguida, na segunda etapa, ocorreu a seleção dos procedimentos abordados em cada grande tópico: septoplastia; cirurgia de catarata; craniotomia; otoscopia; parotidectomia; punção da veia subclávia; cricotireoidostomia; traqueostomia; tireoidectomia; tonsilectomia; toracocentese; ressecção pulmonar; mastectomia e mamoplastia; revascularização do miocárdio; toracotomia; punção venosa da fossa cubital; interrupção do fluxo sanguíneo na artéria braquial após deslocamento do cotovelo; linfadenectomia; reconstrução do ligamento colateral ulnar; liberação do túnel do carpo; enxerto ósseo; artroplastia de quadril; infusão intraóssea; enxerto de veia safena; artroplastia total de joelho.

Após a seleção dos procedimentos, foi realizada a seleção de imagens cadavéricas, do guia de anatomia Asclépio (ROSA ABF, et al. 2011), correlacionadas aos procedimentos cirúrgicos selecionados. Logo após, ocorreu a seleção de artigos científicos com imagens sequenciais dos procedimentos selecionados, seleção de casos clínicos abrangendo os procedimentos cirúrgicos selecionados, e a seleção e adaptação de questões anatômicas relacionadas aos procedimentos cirúrgicos selecionados.

O material de ensino se inicia com a definição do procedimento selecionado, seguido por imagens com especificações nas legendas das regiões de referência ou porção anatômica necessária para a realização 
do procedimento ou técnica cirúrgica. Em seguida, descreveu-se o procedimento com maior detalhamento, utilizando-se de imagens sequenciais e informações adicionais às citadas nas bibliografias básicas. Atualidades sobre as variações do procedimento ou técnicas também foram acrescentadas através da pesquisa de artigos científicos voltados às temáticas. Para finalizar o material, utilizou-se casos clínicos com questões norteadoras acerca do procedimento e/ou estrutura anatômica, permitindo assim, a oportunidade de futuras discussões ao final de cada atividade. As atividades descritas foram reunidas na Figura 01.

Figura 01 - Apostila teórico-prática correlacionando estruturas anatômicas com procedimentos ou técnicas cirúrgicas voltadas à clínica médica.

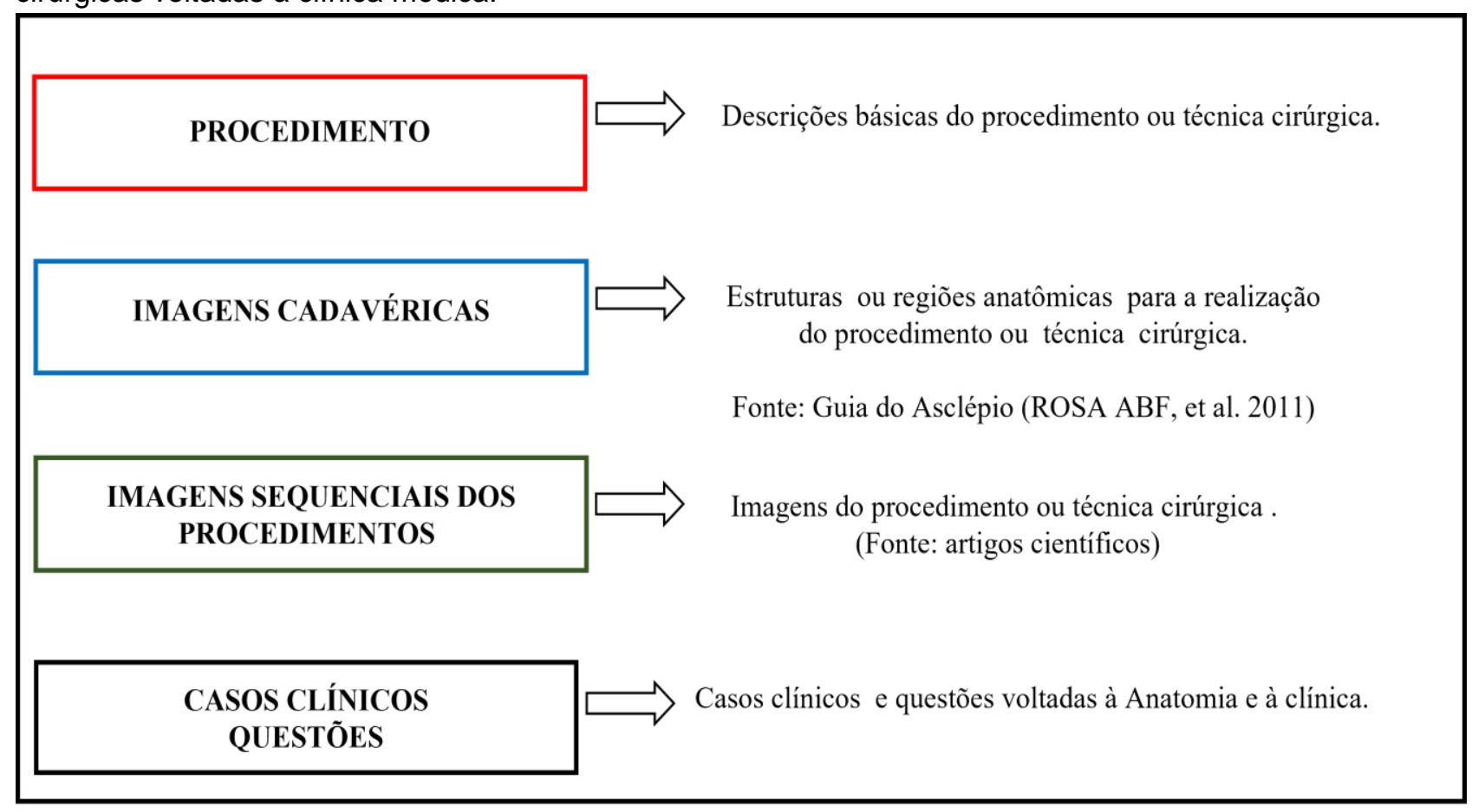

Fonte: Moura JLN, et al., 2021.

\section{DISCUSSÃO}

O estudo da Anatomia Humana é primordial para os cursos da saúde é a sendo considerado de grande importância para a formação de profissionais da saúde, já que o seu entendimento é necessário para que um bom profissional seja formado (MESQUITA GN, et al., 2019). Todavia, o ensino dessa disciplina foi historicamente pautado por uma metodologia passiva em que o aprendiz era submisso e dependente do professor, caracterizando um processo monótono que minimizava a comunicação bilateral e a formação do pensamento crítico (IWANAGA J, et al., 2021).

Diante disso, o desenvolvimento do componente presencial da matéria, com a visualização das estruturas em modelos anatômicos e modelos cadavéricos, se fez primordial ao bom ensinamento, uma vez que é responsável por trazer a interlocução entre a prática e a teoria, gerando questionamentos e promovendo a fixação do conteúdo expositivo. As aulas realizadas no laboratório são significativas em facilitar a aprendizagem devido ao fato de ser um espaço lúdico e interativo (SALBEGO C, et al., 2015). Contudo, a pandemia gerada pelo novo coronavírus e adoção das normativas sanitárias representaram um entrave na aprendizagem da matéria.

Perante tal quadro, foi confeccionado um instrumento de ensino que abordou a sinalização de determinados pontos de referência ou regiões anatômicas fundamentais para a realização de procedimentos ou técnicas utilizadas na prática clínica. Nos livros de Anatomia voltados à Clínica, geralmente encontra-se informações no final de cada capítulo ou tópico sobre procedimentos ou técnicas utilizadas na prática médica. 
No entanto, não trazem em um mesmo tópico correlações direcionadas entre as estruturas anatômicas que são utilizadas como pontos de referência ou vias para os acessos (LONGHURST GJ, et al., 2020).

Muitas vezes, o aluno não consegue correlacionar a importância do estudo de determinadas estruturas ou regiões anatômicas para futuros procedimentos da prática profissional. Tendo isso em vista, foi gerada uma apostila teórico-prática que será utilizada no ensino remoto da disciplina Anatomia Humana e Cirúrgica. A disponibilização de materiais didáticos complementares de forma online refletiu um benefício no ensino da disciplina de Anatomia, segundo estudos realizados no Reino Unido e na República da Irlanda durante o isolamento social provocado pela pandemia de COVID-19 (LONGHURST GJ, et al., 2020).

Dessa forma, para os aprendizes que utilizarão o material, espera-se que seja possível facilitar a absorção do conteúdo, tornando o ensino remoto mais dinâmico e fluido de modo a despertar a curiosidade, bem como o interesse. Por meio da recapitulação das estruturas anatômicas e das imagens cadavéricas, é pretendido assimilar o conteúdo teórico e esquemático com o real, uma vez que as fotografias se sobressaem em relação a desenhos das estruturas corporais, por elucidar a cor, forma e tamanho fidedignos, evitando que ao se deparar em situações práticas (como em laboratórios), quando as atividades presenciais retornarem, 0 estudante enfrente falta de familiaridade (NEMETZ LD,2020).

Por meio da compreensão da Anatomia e do contato, nos primeiros semestres, com discussões que apresentem a importância da correlação anatômica para o estudo de casos como patologias e acidentes, o graduando passa a ser capaz de elaborar métodos para o manejo clínico, favorecendo o direcionamento do estudo e a sedimentação do conteúdo (CINTRA RB, 2017). Outro ponto a ser ressaltado é o entendimento da disciplina ao ser aplicada e não somente sua memorização: fator colocado como necessário para o bom aproveitamento da disciplina (SINHA S, 2014).

Ademais, a problematização da teoria através da correlação cirúrgica, caso clínico e questões pode fomentar no leitor inquietações, desencadeando na busca por maior elucidação do conhecimento, a prática do conhecimento construtivista é baseada em desequilíbrios cognitivos, gerados por conflitos, os quais levam ao raciocínio e tornam o sujeito ativo no processo de aprendizagem (FREITAS, et al., 2019). O processo de absorção do conhecimento ativo deve-se valer de metodologias multissensoriais, incentivando o estudante a participar, relembrar, discutir e traçar formas de reação a situações apresentadas, sejam elas em material didático ou no cotidiano acadêmico (STRINI PJSA, et al., 2020).

Sabe-se que a utilização de metodologias ativas permite uma prática pedagógica modernizada, mais próxima aos alunos. Observa-se, também, que sua utilização permite inúmeras opções de aprendizado, tornando sua aplicação mais vantajosa quando comparada somente ao modelo tradicional de ensino. Assim, para que ocorra a motivação e envolvimento dos discentes, a escolha do método é essencial, já que cada proposta possui diferentes fundamentos, bem como aplicações (SANTOS JW, et al., 2017).

Nesse sentido, a discussão dos casos clínicos permite que o aluno adquira conhecimentos e que os correlacionem com a futura prática médica, uma vez que estudarão e analisarão procedimentos, patologias ou situações que acometem os diferentes tipos de órgãos (COLARES MAM, et al, 2019). O aluno é encorajado a buscar informações para sanar os questionamentos propostos nas atividades, ocorrendo, dessa maneira, uma forma ativa na construção do seu aprendizado (SAVERINO D, 2020).

Segundo Mello JM, et al. (2010), diferentes opções didáticas são proporcionadas quando se utiliza estratégias complementares de ensino como as metodologias ativas. O aluno torna-se o personagem principal e o maior responsável pelo processo de aprendizado, vislumbrando-se assim, o incentivo ao desenvolvimento da capacidade de absorção dos conteúdos de maneira autônoma bem como participativa (MELLO, et al., 2010; KARBASI Z e NIAKAN KALHORI SR, 2020).

O presente relato de experiência descreve o desenvolvimento um material didático anatomoclinico desenvolvido por graduandos do curso de Medicina e uma universidade federal no centro oeste do Brasil. $\mathrm{O}$ desenvolvimento do material permitiu a integração de correlações de determinadas estruturas ou regiões anatômicas com procedimentos ou técnicas empregadas à prática clínica gerando uma apostila teórico-prática contendo textos descritivos, casos clínicos e questões de avaliação acerca da temática proposta. 


\section{AGRADECIMENTOS}

Agradecemos a professora Dra. Daniela Cristina de Oliveira Silva, Professora da Disciplina de Anatomia da Universidade Federal de Uberlândia (ICBIM, UFU), os acadêmicos da Faculdade de Medicina da Universidade Federal de Uberlândia (UFU): Alan Bartasson Ferreira Rosa, Pedro Zerri David e também ao Técnico do Laboratório de Anatomia (ICBIM, UFU) Lázaro Antônio dos Santos, por terem permitido a utilização de imagens cadavéricas obtidas do Asclépio Atlas de Anatomia (http://guiadeanatomia.com/anatomia.html).

\section{REFERÊNCIAS}

1. ALBUQUERQUE JDS, et al. Aprendendo de olhos fechados: ensino da anatomia do coração e vasos da base por meio da identificação tátil. Revista Eletrônica Acervo Saúde, 2020; (49): e3349.

2. CABRAL RH, MASUKO TS. A panorama of medical education in Brazil: Anatomy in focus. Anatomical Record, 2021; 304: 1181- 1184.

3. CASTRO KS, et al. O ensino da anatomia humana através de metodologias ativas de aprendizagem: um relato de experiência. Revista Eletrônica Acervo Saúde, 2021; 13(2): e6176.

4. CINTRA RB. Desafios do ensino da Anatomia Humana em faculdades de Medicina. Revista Cientifica UMC, 2017; 2(1): 1-17.

5. COLARES MAM, et al. Metodologias de ensino de anatomia humana: estratégias para diminuir as dificuldades e proporcionar um melhor processo de ensino-aprendizagem. Arquivos do Mudi, 2019; 23 (3):140-160.

6. FREITAS AS, et al. Percepção acadêmica do processo de ensino e aprendizagem sob metodologias ativas na graduação em fisioterapia de uma universidade pública. Revista Eletrônica Acervo Saúde, 2019; (33): e1232.

7. GOMES VTS, et al. A pandemia da covid-19: repercussões do ensino remoto na formação médica. Revista Brasileira de Educação Médica, 2020; 44(4): e114.

8. IWANAGA J, et al. A review of anatomy education during and after the COVID-19 pandemic: Revisiting traditional and modern methods to achieve future innovation. Clinical Anatomy. 2021; 34:108- 114.

9. KARBASI Z, NIAKAN KALHORI SR. Application and evaluation of virtual technologies for anatomy education to medical students: A review. Medical Journal of Islamic Republic of Iran. 2020; 3:34-163

10. LONGHURST GJ, et al. Strength, weakness, opportunity, threat (SWOT) analysis of the adaptations to anatomical education in the United Kingdom and Republic of Ireland in response to the Covid-19 pandemic. Anatomical Sciences Education, 2020; 13 (3): 301-311.

11. MELLO JM, et al. O Laboratório de Anatomia Humana como espaço não formal de ensino. Arquivos do Mudi, 2010; 14 1/2/3:19-26.

12. MESQUITA GN, et al. Métodos de ensino integrados em monitoria de anatomia e Histologia: um relato de experiência. Revista Eletrônica Acervo Saúde, 2019; (30): e1370.

13. NEMETZ, LD. What You See is What You Understand: How Changes in Dissection Techniques Have Informed Our Understanding of What We Perceive in Anatomy. The FASEB Journal, 2020; 34: 1-1

14. SALBEGO C, et al. Percepções acadêmicas sobre o ensino e a aprendizagem em anatomia humana. Revista Brasileira de Educação Médica. 2015;39(1): 23-31.

15. SANTOS JW, et al. Metodologias de ensino aprendizagem em anatomia humana. Ensino em Re-Vista, $2017 ; 24$ (2): 364-386.

16. SAVERINO D. Teaching anatomy at the time of COVID-19. Clinical Anatomy. 2020; 29:10

17. SILVA JPBM. A história da Anatomia e sua importância no desenvolvimento das ciências farmacêuticas. Dissertação (Mestrado em Ciências Farmacêuticas) -Universidade Lusófona de Humanidades e Tecnologias, Lisboa, $2014 ; 200$ p.

18. SINHA S. How can clinical anatomy teaching be rationalized? Anz Journal of Surgery. 2014;84(11): 805-806.

19. STRINI PJSA, et al. Metodologia ativa em aulas práticas de anatomia humana: A conjunta elaboração de roteiros. Ensino em Re-Vista. 2020; 27 (2): 680-697.

20. ROSA ABF, et al. 2011. In: Guia de Anatomia Humana Asclépio. Uberlândia: Universidade Federal de Uberlândia. Disponível em: http://guiadeanatomia.com/. Acesso em: 10 mar. 2021

21. TALAMONI ACB, BERTOLLI FILHO C. A anatomia e o ensino de anatomia no Brasil: a escola boveriana. História das Ciências da Saúde. 2014;21(4): 1301-1322. 